\title{
Biosurfactants - A New Paradigm in Therapeutic Dentistry
}

\author{
Dr. Ranjitkumar Patil ${ }^{1}$, Saman Ishrat ${ }^{2 *}$, Dr. Akhilanand Chaurasia ${ }^{3}$ \\ ${ }^{1,}{ }^{3}$ Department of Oral Medicine and Radiology, King George Medical University, Lucknow, India \\ ${ }^{2}$ Department of Oral Medicine and Radiology, Rama Dental College, Rama University, Kanpur, India
}

DOI: $\underline{10.36348 / \text { sjm.2021.v06i01.005 }}$ | Received: 08.01.2021 | Accepted: 19.01.2021 | Published: 26.01.2021

*Corresponding Author: Dr. Saman Ishrat BDS, MDS

\section{Abstract}

Biosurfactants are biomolecules with surface active properties, produced mostly by microbes and which offer potential commercial applications. Chemically, biosurfactants can be either glycopeptides, lipopeptides/lipoproteins, fatty acids, phospholipids or neutral lipids, particulate extracellular membranes or polysaccharides conjugated with proteins. Despite of varying chemical composition and molecular weights, all biosurfactants possess the characteristic properties of surface activity, like, lowering surface energy, amphiphilic behaviour towards organic and inorganic solvents. These biomolecules also increase the permeability of and disrupt biomembranes, disrupt biofilms, bind proteins, with bacteriostatic or bactericidal effects. Bacteria may show short-term or long-term tolerance to these effects. In dentistry, biosurfactants offer a vast scope in development and potential use due to their anti-inflammatory as well as antiadhesive activity, immunomodulatory action, antimicrobial applications (antiviral, antitubercular, antibacterial, antifungal), antineoplastic activity and novel uses in gene therapy and drug delivery. The rising death toll in the ongoing COVID-19 crisis is also pushing towards novel avenues of research. Current management of patients is mainly symptomatic but biosurfactants can potentially be both preventive and even curative agents. As the ongoing global environmental, economic and healthcare crises continue to develop, biosurfactants offer hope. Successful commercial use will depend on how well the scientific community and industry leaders clear the bottlenecks in production and supply chain optimisation.

Keywords: Biosurfactants, glycopeptides, Particulate biosurfactants.

Copyright (C) 2021 The Author(s): This is an open-access article distributed under the terms of the Creative Commons Attribution 4.0 International License (CC BY-NC 4.0) which permits unrestricted use, distribution, and reproduction in any medium for non-commercial use provided the original author and source are credited.

\section{INTRODUCTION}

Biosurfactants are biomolecules with surface active properties. Importantly, these are not synthesized chemically but are produced by living cells, which are mostly microbes. This definition does not cover the other common examples of surfactants produced by tissues/organs in higher organisms; like bile salts, which help in emulsification of fats in digestion, or lecithin present in eggs, or surfactant present in lungs that helps in stabilising alveoli and reduces surfaces tension at tissue-air interface for exchange of gases. As a field of study, then, the study of biosurfactants focuses on those compounds with potential commercial use that are produced by microorganisms.

In 1949, FG Jarvis and MJ Johnson working at Department of Biochemistry, University of Wisconsin, reported the isolation of a glycolipid, produced by Pseudomonas aeruginosa, which they noted, was bacteriostatic to a strain of Mycobacterium tuberculosis (H37 Rv), in a concentration of $0.5 \mathrm{mg} / \mathrm{mL}$ of culture medium. They elucidated it to consist of L-Rhamnose and normal 1-ß-hydroxydecanoic acid and found its crystals to be acidic and highly soluble in most organic solvents and partially soluble in water [1]. This effectively spurred activity in the field of biosurfactants. Today, biosurfactants find uses in fields as diverse as petrochemicals, agriculture, commercial food industry, cosmetics, and pharmaceuticals, among others. However, synthetic surfactants continue to be popular owing to the competent pricing and high production costs and low yield are still formidable challenges to making biosurfactants mainstream in industry. In medicine, these new class of compounds hold promise in treating infections, in wound healing through antiinflammatory and immunomodulatory activity, and in drug delivery. These applications are also useful in the practise of dentistry. Additionally, biosurfactants show promise in osseointegration of implants a local drug delivery.

\section{Parallel Developments in World}

In 1968, Barrett, McGehee Jr. and Finland reported that 21 out of 22 strains of Staphylococcus aureus obtained from patients at their hospital showed resistance to penicillins, methicillin and cephalosporin. They also noted infection of these strains to spread from 
patient to patient and nasopharyngeal carriage of resistant strains [2]. The development of penicillin, streptomycin, chloramphenicol and tetracycline between 1945-55 was a breakthrough in medicine. Yet, within a decade of ushering in the age of antibiotics with these drugs, resistant strains had begun to emerge. Mentioned at the beginning of this article, the biosurfactant discovered by Jarvis and Johnson in 1949, eight years after the term antibiotic was coined by Selman Waksman, was not a major contender in this developing scenario, until this time. It was as yet an academic curiosity.

Eventually, reports started coming in about the presence of high levels of antibiotics in rivers, from almost all countries of the world. Hirsch et al. described about 18 antibiotic substances in aquatic environment in 1999. These included sulfamethoxazole, roxithromycin, degraded products of erythromycin, penicillins and macrolides. At that time except for two sites in Germany, no site showed significant contamination and the authors concluded intake from veterinary use was of minor importance [3]. Within two decades, this experience underwent a major upheaval with antibiotics reported in almost all major rivers of the world, with reports coming in from Japan, China, Europe, and Kenya among others [4-7].

Fifty years after the first report of antibiotic resistance in a hospital setting, Otto Cars and Per Nordberg of Swedish Institute for Infectious Disease Control, published about the looming threat of antibiotic resistance, which was widespread enough to upend medical advances in infection control, with Thailand and India showing about $80-90 \%$ resistance to more than one antibiotic. They considered it a system failure, with multiple causes like breakdown of relationship between pharmaceutical industry and the community, overcrowding, rampant use of antibiotics in commercial food production, etc [8]. The full scale of this crisis was reported in 2014, when the World Health Organisation (WHO) published its report Antimicrobial Resistance: Global Report on Surveillance, with data pooled from 114 countries. Seven bacteria that caused serious infections were documented to be resistant even to those antibiotics which were used as a last resort in all surveyed countries [9]. At present, six years later, this is an ongoing crisis. In 2015, the United Nations (UN) forwarded a policy change advocating Sustainable Development Goals (SDG). The 17 goals address climate change, renewable energy, and coordinated environmental and socioeconomic monitoring and modelling.

Bioremediation is removal of contaminants from environment. This could range from treating polluted groundwater to removing oil spills. Currently, the bulk of bioremediation is done with the help of synthetic chemicals. However, in accordance with SDG, biosurfactants are increasingly appearing as a viable alternative. These can also be used in industry where phase changes are an important part of production, like solubilising agents and emulsion forming or breaking compounds. Rhamnolipid biosurfactants obtained from P. aeruginosa UG2 removed both aliphatic and aromatic hydrocarbons in unsaturated soil columns and compared favourably with the chemical surfactant Triton X-100 [10]. Sun, Wang, Zang et al. recently isolated a glycolipid biosurfactant $\mathrm{S} 5$, from a $\mathrm{P}$. aeruginosa strain that they isolated from coking wastewater. $\mathrm{S} 5$, at a CMC of $96.5 \mathrm{mg} / \mathrm{L}$, reduced surface tension from $72.2 \mathrm{mN} / \mathrm{m}$ to $29.6 \mathrm{mN} / \mathrm{m}$ and degraded high molecular weight (HMW) polycyclic aromatic hydrocarbons (PAH) in sludge phase; while showing stability at wide variations of $\mathrm{pH}$ and salinity [11]. Sophorolipids are already in a significant commercial yield while glycolipids, rhamnolipids and mannosylerythritol lipids need development. Unlike their chemical counterparts, biosurfactants can be tailored specifically. Thus, biosurfactants offer a sustainable alternative to bulk chemical commercial laundry detergents or emulsifiers/demulsifiers. COVID19 is a disease caused by Severe Acute Respiratory Syndrome- Coronavirus-2 (SARS-CoV-2). The rising death toll in this ongoing multipronged crisis pushes us towards novel avenues of research. At its simplest, the idea is to directly disrupt the lipid membrane of the SARS-CoV-2, with biosurfactants and damage the integrity of viral proteins and genetic material, selectively avoiding damage to host membranes. High specificity, low toxicity and the additional advantage of sustainability make this a promising approach. Current treatment of SARS-CoV-2 is mainly symptomatic but biosurfactants offer hope in being both preventive and even curative agents in management of the pandemic [12].

In the light of all these parallel developments, the use of biosurfactants impacts the medical and dental professions both, in multiple ways. Once we have understood the broad concept of what biosurfactants are and how they interact with a physical or a biological medium, we can then begin to consider the plethora of applications available for use of biosurfactants in dentistry.

\section{PROPERTIES OF BIOSURFACTANTS}

\section{A. Physical properties}

Surface: It is taken to be the outermost boundary of a substance, in any phase of the matter. Surface phenomenon, then, are vital in industries, where miscibility or solvency of different substances are crucial. It has often been suggested by physicists that surface free energy is a better determinant of surface phenomenon than surface tension but for the sake of laity we shall explain physical properties of biosurfactants in terms of surface tension.

Surface Tension: Particles in the bulk of a substance exert a thermodynamic force on each other. 
Within the substance, this force on an internal particle more or less cancels out, being the same in all directions but at the surface it leads to a net pull inwards, on the surface molecules. This gives rise to surface tension. Surface tension is measured in newtons per metre $(\mathrm{N} / \mathrm{m})$.

Interfacial tension: Just that unlike surface tension which exists due to cohesive forces between the molecules of a liquid in contact with a gaseous phase, interfacial tension exists between two substances due to adhesive forces between liquid phase of one and either (solid/liquid/gas) phase of another substance. Lowering or increasing contact angles between substances to effect change of surface tension or interfacial tension therefore is a major deciding factor between wettability, miscibility, emulsification, floatation etc. and affects important industrial processes with major economic implications. Currently, most industries use chemical surfactants.

Critical micelle concentration (CMC): commonly measured in $\mathrm{mg} / \mathrm{L}$, is the minimum amount of concentration of the surfactant required for a unit lowering of surface energy [7].

Like their chemical counterparts, biosurfactants too have amphiphilic behaviour showing affinity for both water-based and oil-based solvents and theoretically therefore can be similarly employed in industrial applications. For example Rhamnose lipids obtained from $\mathrm{P}$. aeruginosa lower the surface tension of water from $72 \mathrm{mN} / \mathrm{M}$ to $27 \mathrm{mN} / \mathrm{m}$ at a $\mathrm{CMC}$ of $150 \mathrm{mg} / \mathrm{L}$ [13]. Sophorolipids reduce the same to 35 to $60 \mathrm{mN} / \mathrm{m}$ at a CMC of $5-80 \mathrm{mg} / \mathrm{L}$, and even up to $26 \mathrm{mN} / \mathrm{m}$ under special conditions [14].

\section{Concepts of Biosurfactant Mechanism}

\section{A. Biofilms}

A natural propensity to form biofilms is a universal feature of bacteria. Biofilms are multicellular communities of bacteria existing in an extracellular matrix secreted by the member bacteria themselves. The most famous example of biofilm in dentistry is dental plaque. It has been proposed that formation of biofilm is triggered by extracellular signals. Escherichia coli, Staphylococcus aureus, Pseudomonas aeruginosa and Bacillus subtilis are medically important species of bacteria that form biofilms. Initially, the bacteria adhere to an inert surface through purely physico-chemical forces, and later they develop molecular adhesion to the surface, after which the biofilm matures and later microbes detach from the matured biofilm to establish another biofilm at another site. This is the general course of maturing of biofilms [15]. The formation of biofilm can lead to serious problems in medical water supply lines, in-dwelling catheters, implants and valves. Biofilms are notoriously difficult to remove because cells are embedded in a polymer matrix resistant to degradation by common methods.

\section{B.Quorum Sensing}

It is the phenomenon of change in gene expression as a response to cell population density. Bacteria use this to effect change in a variety of physiological activities like symbiosis, virulence, biofilm formation among others. For this purpose, the bacteria use chemicals called autoinducers, which modify gene expression and can alter the behaviour of the entire community of bacterial species in a specific environment, showing a behaviour somewhat parallel to hormones in higher organisms. Gram-positive bacteria use processed oligopeptides and the gram-negative bacteria use acylated homoserine lactones as autoinducers. Quorum sensing may have been an important step towards evolution of multicellular living organisms [16].

\section{C.Interaction with Biological Membranes}

Biosurfactants increase permeability of and disrupt biomembranes: Rhamnolipid-biosurfactant increases permeability of bacterial cell membranes. Sotirova et al. obtained a surface-active complex PS-17 (named so because it was produced by Pseudomonas sp. PS-17 strain). It consisted of an extracellular biopolymer polysaccharide and esters of rhamnose and 3-oxydecanic acid. PS-17 formed stable, highly dispersed emulsions with lowered surface tension and at a concentration of $0.05 \%$, completely inhibited the growth of the gram-positive Bacillus subtilis 168. PS-17 however, failed to disrupt the cell membranes and protein production of gram-negative bacteria studied i.e. P. aeruginosa and E. coli [17].

This difference in response of gram-negative and gram-positive bacteria may be due to the very nature of their membranes. The gram-negative bacteria show organic solvent tolerance by reducing the fluidity of cell membrane, mainly by two mechanisms: shortterm tolerance, where they convert unsaturated membrane fatty acids from cis- to trans- form; and a long-term tolerance by increasing the saturated fatty acids in the phospholipids of cell membrane or by increasing the amount of long chain fatty acids. The gram-positive bacteria Staph. haemolyticus studied by Nielsen et al., however, showed a completely opposite response to membrane fluidity, in that it increased the permeability of membrane, in response to organic solvents, by increasing the amount of anteiso fatty acids and decreased straight-chain fatty acids[18].

Sophorolipids disrupt biofilms and have a bactericidal effect at concentrations of $5 \% \mathrm{v} / \mathrm{v}$. In a study done by Mayri et al., both simple and mixed cultures of Bacillus subtilis BBK006 and Staphylococcus aureus ATCC 9144, which are grampositive bacteria; and the Gram-negative Cupriavidus necator ATCC 17699, were observed. Disruption of biofilms and inhibition of growth were noted in the interaction of biosurfactants with the cell membranes of these bacteria [19]. This year in a study by Olivia et al 
showed that a biosurfactant produced by P. aeruginosa disrupted model membranes consisting of dimyristoylphosphatidylserine by changing the structure and phase behaviour by increasing hydration [20].

D.Protein Binding: A glycolipid biosurfactant Mel-A shows high protein binding affinity for human immunoglobulin G (HIgG). Acetyl groups of sialic acid are postulated to be the reason for this binding and MelA with two acetyl groups on its mannose moiety shows this biologic affinity. It also shows efficient eluting of $\mathrm{HIgG}$, which is a promising development in the fields of immunodiagnostics, mapping of epitopes and in therapeutics [21].

\section{TYPES OF BIOSURFACTANTS}

\section{A. Based on chemical composition}

1. Glycolipids- most biosurfactants under study fall in this group. In these a sugar moiety (glucose, rhamnose, galactose, glucuronic acid, mannose) is conjugated with either a long chain aliphatic acid or a hydroxyaliphatic acid through ester linkages. This includes rhamnolipids, sophorolipids, trehalolipids, etc.

2. Lipopeptides and Lipoproteins- contain a lipid conjugated to a polypeptide chain. Lipopeptide from Bacillus sp. Like polymyxin and cyclic decapeptides (gramicidin) belong to this category, both of which have antibiotic effect.

3. Fatty acids, phospholipids and neutral lipidsproduced by bacteria and yeast strain grown on $n$ alkane containing medium, these are complex fatty acids with hydroxyl groups and alkyl branches. Corynomuolic acid is one such biosurfactant. Phosphatidylethanolamine from Acinetobacter sp. is another.

4. Polymeric biosurfactants- like emulsan, liposan, mannoprotein are polysaccharides conjugated to proteins

5. Particulate biosurfactants: are extracellular membranes which microemulsify hydrocarbons and help in uptake of the same by bacterial cell.

\section{B. Based on molecular weight}

1. High molecular weight- commonly called bioemulsan, these are complex mixtures of extracellular polymers like polysaccharides, proteins, lipopolysaccharides, lipo-proteins. They show high efficiency in emulsifying oil in water, high substrate specificity, and a low CMC.

2. Low molecular weight- generally glycolipids or lipopetides, these are efficient in lowering surface energy at air-water interface.
APPLICATIONS IN DENTISTRY
A. Anti-inflammatory activity
B. Antiadhesive activity
C. Immunomodulatory activity
D. Antimicrobial applications
1. Antiviral activity

2. Antitubercular activity

3. Antibacterial activity

4. Antifungal activity

E. Antineoplastic activity

F. Gene Therapy

G. Drug delivery

A. Anti-Inflammatory and Anti-Allergic Activity: In dentistry, the role of A-delta and C fibres in pain relay pathway has a very important significance. The dental pulp being encased in a rigid dentin layer does not often have recourse for oedematous swelling to expand, and therefore, inflammatory disorders either elicit a pain response from patient in acute phase of inflammation or lead to necrosis of pulp in chronic one. The glycolipid biosurfactant Mannosyl-Erythritol Lipids (MELs) have been shown to affect the important intracellular signalling pathways that involve MAP kinases and $\mathrm{Ca}^{2+}$. MELs were shown to inhibit increase in calcium and phosphorylation of MAP kinases as well as inhibit secretion of mediators of inflammation, like leukotriene $\mathrm{C}_{4}$ and TNF- $\alpha$ from mast cells, thereby having anti-inflammatory effect and anti-allergic effect as well [22].

An important area of research currently is the role of this application in SARS-CoV-2 infection, where interaction between the virus and host cells, especially in lungs, triggers a cytokine storm. Sophorolipids obtained from C. bambicola have been shown to decrease lung inflammation by decreasing IgE, IL-6, and gene expression of TLR-2. This reduction of gene expression of inflammatory cytokines holds promise in both SARS-CoV-2 infection and even for immunomodulation in chronic inflammatory conditions for biosurfactants to be used as novel therapeutic agents [23].

\section{B. Antiadhesion Activity}

The ability of biofilms to adhere to surfaces is a key factor in survival of resistant strains of microbes. The inherent amphiphilic nature of biosurfactants can be used to disrupt the integrity of these biofilms. Once the bacterial biofilm has been disrupted, biosurfactants and innate immunity can destroy the pathogenic bacteria or keep their numbers in check. Adsorption of crude biosurfactant obtained from Streptococcus mitis BMS, increased repulsion of plaque forming Strep. mutans strains HG 1025 and ATCC 25175, on both bare and pellicle-coated enamel [24]. Bacillus tequilensis $\mathrm{CH}$, a halophile isolated from Lake Chilika in India, produces a lipopeptide biosurfactant which can inhibit biofilm formation of pathogenic E. coli and Strep. mutans, at as low CMC as $50 \mu \mathrm{g} / \mathrm{mL}[25]$. Biosurfactant CV8LAC obtained from a Lactobacillus sp. isolated from cabbage showed inhibitory activity against two pathogenic strains of C. albicans. It disrupted biofilm formation of strain DSMZ 11225 by $81 \%$ at a CMC of $19.95 \mu \mathrm{g} / \mathrm{mL}$. A similar effect was seen on biofilm 
adherence of Listeria monocytogenes, Salmonella arizonae, E. coli and S. aureus on polystyrene and Listeria monocytogenes on stainless steel [26]. Biosurfactants produced by $\mathrm{P}$. aeruginosa UCP 0992(PB), Bacillus metylotrophicus UCP 1616(BB) and Candida bombicola URM 3718(CB) were evaluated for use in toothpaste formulation with chitosan. The formulated toothpastes were noted to be non-toxic, and had a $\mathrm{pH}$ of 9 , spreading capacity between $8-17 \mathrm{~mm}$ and $63-95 \%$ foaming. Both $\mathrm{PB}$ and $\mathrm{CB}$ showed additive effect with chitosan against Streptococcus mutans and BB showed negligible effect. This property of preventing plaque formation shows promise in commercial toothpaste formulation [27].

A major concern in dental implantology is development of inflammation around the implant (periimplant mucositis, periimplantitis), which interferes with osseointegration of the implant. The rhamnolipid biosurfactant R89BS, produced by P. aeruginosa 89, at a CMC of $4 \mathrm{mg} / \mathrm{mL}$, when coated on titanium discs, inhibited biofilm formation of S. aureus by more than $90 \%$ and almost $70 \%$ for S. epidermidis, at 24 hours. Interestingly, this inhibition of biofilm formation was independent of the surface morphology of the implant [28].

\section{Immunomodulatory Activity}

Rhodococcus, a genus of actinobacteria produces several trehalolipid biosurfactants, of which Trehalose Dimycolate (TDM) shows granulomatogenic activity by directly inducing chemotaxis of macrophages and enhancing secretion of proinflammatory cytokines. The ability to induce cytokines, exert an anti-tumour effect through TNFalpha and induce angiogenesis is some other important immunomodulatory properties of TDM [29].

\section{Antimicrobial Applications \\ 1. Antiviral Activity}

Human Herpes Virus infections are commonly seen in dental clinics, with Herpes Simplex Virus 1 and 2 (HSV-1, HSV-2) commonly causing blistering diseases of oral mucosa. The other virus of importance in a dental setting is HIV 1 and 2. Most patients need management of conditions arising from an infection by these viruses and the bulk of such management at present is done with supportive treatment or sometimes antiviral drugs [30]. Biosurfactants open a possibility of targeted virucidal drugs with lower cytotoxicity profile. A cyclic lipopolypeptide biosurfactant obtained from Bacillus subtilis, Surfactin exhibited showed biphasic virus inactivation, at $\mathrm{CMC} 25 \mathrm{mM} / \mathrm{L}$ by disrupting viral lipid membrane. Destruction of enveloped viruses like HSV-1 and 2 and retroviruses was more efficient than of those without such an envelope. Although, Surfactin demonstrated low in vivo toxicity in mice, it caused haemolysis and inhibited fibrin clot formation. In vitro cytotoxic evaluation showed inhibition of mammalian cell proliferation at a CMC above $20-40 \mathrm{mM} / \mathrm{L}$, in a one cell passage period (3-7days), whereas it inactivated susceptible viruses at a lower CMC within a few minutes [31]. Pumilin, an analogue of Surfactin, obtained from B. pumilus showed $50 \%$ inhibition of $\mathrm{HSV}-1$ at CMC of 3.4 to $6.4 \mathrm{mM} / \mathrm{L}$ [32]. Di-acylated ethyl ester of Sophorolipid from Candida bombicola ATCC 22214, showed good antiviral activity against HIV [33].

2. Anti-Tubercular Activity-Mycobacterium tuberculosis (Mtb) spontaneously forms pellicle biofilm, which hosts more drug resistant Mtb bacteria than those in dispersed culture. Population enrichment of resistant strains in this biofilm is most likely due to selective selection of cells with intrinsic drug tolerance [34]. Rhamnolipid biosurfactants obtained from P. aeruginosa ATCC 9027 grown on phosphate limited medium showed activity against Mycobacterium aurum, which is used as a surrogate for M. tuberculosis. On culture media with supernatant Rhamnolipid at a CMC of $3.954 \mathrm{~g} / \mathrm{L}$, zone of clearance was noted to be $45 \mathrm{~mm}$ [35].

3. Anti-Bacterial Activity-In dentistry, controlling plaque formation is vital to the success of most dental procedures. Lactobacillus rhamnosus derived biosurfactant inhibits biofilm formation of Streptococcus mutans by downregulating the expression of $\mathrm{gtfB} / \mathrm{C}$ and $\mathrm{ftf}$ genes that help in synthesis of extracellular glucans- an important constituent of dental plaque [36]. Within the oral cavity, bacteria can be either in the biofilm community or they may be present in a planktonic one. Data on minimum inhibitory concentration of biosurfactants and which uses NMR spectroscopy to characterise biosurfactants used in studies on oral microflora is still in developing stages. Cyclic lipopeptides produced by Pseudomonas spp. Show antibiotic response to root-pathogenic microfungi [37]. In another study, the minimum inhibitory concentration of sophorolipids for Staph aureus ATCC29737 , was calculated to be $400 \mu \mathrm{g} / \mathrm{mL}$ and behaved synergistically with tetracycline. Similarly, the sophorolipid-cefaclor combination against E. coli ATCC 8739 showed $98 \%$ destruction of pathogen colony in 4 hours whereas cefaclor alone took 6 hours for the same and sophorolipid alone had no inhibitory effect even at concentrations upto $1000 \mu \mathrm{g} / \mathrm{mL}$. Damage to cell membrane integrity is the observed mechanism of action [38]. Rhamnolipid obtained from Pseudomonas aeruginosa OBP1 showed effective antibacterial activity by increasing the permeability of cell membrane, at concentrations above $\mathrm{CMC}$, against Staphylococcus aureus MTCC 3160 and Klebsiella pneumoniae MTCC 618[39]. Glycolipid biosurfactant obtained from Lactobacillus lactis and Bacillus licheniformus showed cidal activity against multiple strains of drug resistant E. coli and Methicillin Resistant Staph aureus (MRSA) [40].

4. Anti-Fungal Activity -Fungal infections of oral mucosal surfaces and dental prostheses are a 
common concern in dental practice. Current method of management with multiple drugs and topical treatment with antifungal agents calls for further research into more efficient and cost-effective preventive measures for the same.

The preventive anti-adhesion activity of biosurfactants against Candida albicans biofilm showed a significant reduction in biofilm cell population than on using chlorhexidine. Amphiphilic disruption of microbial adhesion on discs of silicon and acrylic resin; and compatibility with epithelial and fibroblast cells were noted, when biosurfactants obtained from biofilms of endophytes from Robinia pseudoacacia and Nerium oleander were infected with C. albicans[41].

5.Antineoplastic Activity-In 2015, Kuo, Lin and Chen, reported that the lipopeptide biosurfactant obtained from Bacillus amyloliquefaciens BACY1 (BLE) inhibited proliferation in human oral squamous cell carcinoma lines SCC4 and SCC25. BLE effectively prolonged the sub-G1 phase and upregulated Bax gene expression and caspase 3, which in turn enhanced apoptosis in cancer cell lines and inhibited their proliferation [42].

In a study on human esophageal cancer cell lines KYSE 109 and KYSE 450, to evaluate effect of pure Sophorolipid molecules, it was found that diacetylated lactonic sophorolipid inhibited cell division at CMC of $30 \mu \mathrm{g} / \mathrm{mL}$. Acidic sophorolipid showed no significant antineoplastic activity [43]. A halophilic species of bacteria, Halomonas sp. BS4 isolated from Thamaraikulam solar salt works in India, yielded a biosurfactant, which at a CMC of 2.5 microgram per litre, and suppressed the proliferation of cells of mammary epithelium by $46.77 \%$ [44]. In vitro antitumour activity of the glycolipid Trehalose Lipid Tetraester (THL), which is obtained from Nocardia farcinica BN26, was studied on cancer cell lines BV173, SKW-3, among others. Cytotoxicity of THL to malignant cell lines was noted to be incubation dependent in BV-173 and concentration dependent in SKW-3. Additionally, the effect on normal human cell lines was only weakly cytotoxic [45].

\section{E. Gene Therapy}

Gene therapy relies heavily on viral vectors to edit genes of choice. Viral vectors, especially adenoviral ones, carry a risk of immunogenicity and the use of retrovirus integration into human DNA is fraught with the risk of inducing oncogenesis [46]. As such, research is now turning towards improving viral vectors and exploring non-viral vectors for editing DNA sequences. Novel non-viral agents of transfection include bacteria, virus-like particles (VLPs), erythrocyte ghosts and exosomes.

Gene transfection mediated by cationic liposomes rose dramatically with use of MEL-A.
Liposomes containing cholesterol conjugated Ldioleoylphosphatidylethanolamine (DOPE) along with a biosurfactant like MEL-A or B-sitosterol- $\beta$-D-glucoside, exhibit higher efficiency of gene transfection [47].

\section{F. Drug delivery systems}

Glycolipids tend to organise on their own into supramolecular structures, which may exist as either of the two phases: hexosome, which is an inverted hexagonal, or cubosome, which is a reversed bicontinuous cubic phase. This aqueous nanodispersion can carry Active Pharmaceutical Ingredient (API) like, indomethacin, and cyclosporine-A, irrinotecan, and even insulin. Supramolecular organisation depends on the balance between curvature elasticity and the geometric frustration (as atoms, with conflicting interatomic forces, stick to available positions in lattice formation, thereby leading to complex geometric structures, even as attempting to achieve the simplest possible shape) of the constituents. Interestingly, using glycolipids the nanostructured aqueous dispersions formed showed stability for several months, and in varying strengths of salinity. This opens potential applications for glycolipid biosurfactants as selfassembled drug delivery systems [48]. A nanoemulsion of doxorubicin with eucalyptus essential oil and surfactin was demonstrated to have physical and chemical stability under extreme $\mathrm{pH}$ (3-9), at temperatures up to $45^{\circ} \mathrm{C}$ under an external shearing force of $10,000 \mathrm{rpm}$. It showed sustained release of API for up to 24 hours, approximately 35 times slower release kinetic rate and ten times lower minimum inhibitory concentration than free doxorubicin[49].

Formation of calcium liquid crystal (CLC) of hydroxyapatite pyrophosphate, leads to a stable, microporous spherical structure when egg-white derived ovalbumin is used as a biosurfactant. This helps in protein mediated biomineralisation through two steps. First, protein chains change configuration and CLCs appear in aqueous solution. Next, adsorption of these small spherical CLCs occur on available surface due to interfacial tension between vesicle and water surface. As such, this biomaterial holds promise for bone implantations, for coating roots of teeth and for artificial joints [50].

\section{Current Challenges and Future Research}

Biosurfactants have the advantage of being more efficient, selective, and stable over a wide range of $\mathrm{pH}$, temperature and salinity, than the chemical surfactants. Glycolipids continue to be the most commonly studied biosurfactants, even as everyday a plethora of other novel biosurfactants are discovered and are under study at various parts of the world. Biosurfactants are increasingly required in multiple industries like cosmetics, pharmaceuticals, healthcare, paper processing, coal and metal mining, among others. However, commercialisation of biosurfactants hits a roadblock at cost effective production where synthetic 
chemicals already have established chain of production and use. Development of rapid methods of identification of microorganisms, that produce biosurfactants, with standard screening and evaluation, is in the pipeline. The underlying influence of genetics has been recognised in biosurfactant production genes that synthesize surfactin in Bacillus sp. like sfp, srfA, and comA have been identified and their roles studied. Similarly, gene for synthesis of Rhamnolipid synthesis rhlAB and genes that regulate it i.e. rhll and rhlR have been characterised and their expression has been studied in heterologous hosts.

Biosurfactants meet industry standards in bioremediation and play their largest commercial role in this category, followed by pharmaceutical applications. As research progresses, generation of biosurfactants at competent pricing may begin with the use of agricultural and industrial waste utilisation as substrate for microbial growth, development of cost-effective continuous recovery and safe and effective biotransformation. Process optimisation to make the best use of culture medium and to provide essential factors for growth of culture organisms is vital to commercial production. Additionally, most biotechnological products including biosurfactants, hit a roadblock at cost effectiveness of recovery of product. Downstream costs could be almost $60 \%$ of total production costs and developing fast, effective and cheap methods of purification would be a step in the right direction. A curious problem in the development of biosurfactant as an alternative to synthetic chemicals currently in use in industry, is the almost a counterproductive reality that purification of biosurfactants itself requires the use of environmentally toxic solvents like acetone, methanol, chloroform. Recently, biosurfactants from Rhodococcus sp. have been purified commercially with cheap and relatively less toxic solvent, namely, methyl tertiary-butyl ether (MTBE). Using recombinant microorganisms, which improve yield and also enable better product characteristics, is another promising area of research in commercial production of these biomaterials.

Biosurfactants offer an alternative to the synthetic chemical surfactants in diverse industries. As the ongoing global environmental, economic and healthcare crises continue to develop, this class of biomaterials offers a hope of sustainability. Successful commercialisation will depend on how well the scientific community and industry leaders clear the bottlenecks in production and supply chain optimisation.

\section{REFERENCES}

1. Jarvis, F. G., \& Johnson, M. J. (1949). A glycolipide produced by Pseudomonas aeruginosa. Journal of the American Chemical Society, 71(12), 4124-4126.
2. Barrett, F. F., McGehee Jr, R. F., \& Finland, M. (1968). Methicillin-resistant Staphylococcus aureus at Boston City Hospital: bacteriologic and epidemiologic observations. New England Journal of Medicine, 279(9), 441-448.

3. Hirsch, R., Ternes, T., Haberer, K., \& Kratz, K. L. (1999). Occurrence of antibiotics in the aquatic environment. Science of the Total environment, 225(1-2), 109-118.

4. Murata, A., Takada, H., Mutoh, K., Hosoda, H., Harada, A., \& Nakada, N. (2011). Nationwide monitoring of selected antibiotics: distribution and sources of sulfonamides, trimethoprim, and macrolides in Japanese rivers. Science of the total Environment, 409(24), 5305-5312.

5. Li, X., Shi, H., Li, K., Zhang, L., \& Gan, Y. (2014). Occurrence and fate of antibiotics in advanced wastewater treatment facilities and receiving rivers in Beijing, China. Frontiers of Environmental Science \& Engineering, 8(6), 888894.

6. Johnson, A. C., Keller, V., Dumont, E., \& Sumpter, J. P. (2015). Assessing the concentrations and risks of toxicity from the antibiotics ciprofloxacin, sulfamethoxazole, trimethoprim and erythromycin in European rivers. Science of the Total Environment, 511, 747-755.

7. Kimosop, S. J., Getenga, Z. M., Orata, F., Okello, V. A., \& Cheruiyot, J. K. (2016). Residue levels and discharge loads of antibiotics in wastewater treatment plants (WWTPs), hospital lagoons, and rivers within Lake Victoria Basin, Kenya. Environmental monitoring and assessment, 188(9), 532.

8. Cars, O., \& Nordberg, P. (2005). Antibiotic resistance-The faceless threat. International Journal of Risk \& Safety in Medicine, 17(3, 4), 103-110.

9. World Health Organization. (2014). Antimicrobial resistance global report on surveillance: 2014 summary (No. WHO/HSE/PED/AIP/2014.2). World Health Organization.

10. Scheibenbogen, K., Zytner, R. G., Lee, H., \& Trevors, J. T. (1994). Enhanced removal of selected hydrocarbons from soil by Pseudomonas aeruginosa UG2 biosurfactants and some chemical surfactants. Journal of Chemical Technology \& Biotechnology: International Research in Process, Environmental AND Clean Technology, 59(1), 5359.

11. Sun, S., Wang, Y., Zang, T., Wei, J., Wu, H., Wei, C., ... \& Li, F. (2019). A biosurfactant-producing Pseudomonas aeruginosa S5 isolated from coking wastewater and its application for bioremediation of polycyclic aromatic hydrocarbons. Bioresource technology, 281, 421-428.

12. Smith, M. L., Gandolfi, S., Coshall, P. M., \& Rahman, P. K. (2020). Biosurfactants: a Covid-19 perspective. Frontiers in Microbiology, 11. 
13. Lang, S. (2002). Biological amphiphiles (microbial biosurfactants). Current Opinion in Colloid \& Interface Science, 7(1-2), 12-20.

14. Lourith, N., \& Kanlayavattanakul, M. (2009). Natural surfactants used in cosmetics: glycolipids. International journal of cosmetic science, 31(4), 255-261.

15. Busscher, H. J., \& Van der Mei, H. C. (1997). Physico-chemical interactions in initial microbial adhesion and relevance for biofilm formation. Advances in dental research, 11(1), 2432.

16. Miller, M. B., \& Bassler, B. L. (2001). Quorum sensing in bacteria. Annual Reviews in Microbiology, 55(1), 165-199.

17. Sotirova, A. V., Spasova, D. I., Galabova, D. N., Karpenko, E., \& Shulga, A. (2008). Rhamnolipidbiosurfactant permeabilizing effects on grampositive and gram-negative bacterial strains. Current microbiology, 56(6), 639-644.

18. Nielsen, L. E., Kadavy, D. R., Rajagopal, S., Drijber, R., \& Nickerson, K. W. (2005). Survey of extreme solvent tolerance in gram-positive cocci: membrane fatty acid changes in Staphylococcus haemolyticus grown in toluene. Applied and environmental microbiology, 71(9), 5171-5176.

19. De Rienzo, M. A. D., Banat, I. M., Dolman, B., Winterburn, J., \& Martin, P. J. (2015). Sophorolipid biosurfactants: possible uses as antibacterial and antibiofilm agent. New biotechnology, 32(6), 720-726.

20. Oliva, A., Teruel, J. A., Aranda, F. J., \& Ortiz, A. (2020). Effect of a dirhamnolipid biosurfactant on the structure and phase behaviour of dimyristoylphosphatidylserine model membranes. Colloids and Surfaces B: Biointerfaces, 185, 110576.

21. Im, J. H., Yanagishita, H., Ikegami, T., Takeyama, Y. I., Idemoto, Y., Koura, N., \& Kitamoto, D. (2003). Mannosylerythritol lipids, yeast glycolipid biosurfactants, are potential affinity ligand materials for human immunoglobulin G. Journal of Biomedical Materials Research Part A: An Official Journal of The Society for Biomaterials, The Japanese Society for Biomaterials, and The Australian Society for Biomaterials and the Korean Society for Biomaterials, 65(3), 379-385.

22. Morita, Y., Tadokoro, S., Sasai, M., Kitamoto, D., \& Hirashima, N. (2011). Biosurfactant mannosylerythritol lipid inhibits secretion of inflammatory mediators from RBL-2H3 cells. Biochimica et Biophysica Acta Subjects, 1810(12), 1302-1308.

23. Subramaniam, M. D., Venkatesan, D., Iyer, M., Subbarayan, S., Govindasami, V., Roy, A., ... \& Vellingiri, B. (2020). Biosurfactants and antiinflammatory activity: A potential new approach towards COVID-19. Current opinion in environmental science \& health.
24. Van Hoogmoed, C. G., Dijkstra, R. J. B., Van der Mei, H. C., \& Busscher, H. J. (2006). Influence of biosurfactant on interactive forces between mutans streptococci and enamel measured by atomic force microscopy. Journal of dental research, 85(1), 5458.

25. Pradhan, A. K., Pradhan, N., Mall, G., Panda, H. T., Sukla, L. B., Panda, P. K., \& Mishra, B. K. (2013). Application of lipopeptide biosurfactant isolated from a halophile: Bacillus tequilensis $\mathrm{CH}$ for inhibition of biofilm. Applied biochemistry and biotechnology, 171(6), 1362-1375.

26. Fracchia, L., Cavallo, M., Allegrone, G., \& Martinotti, M. G. (2010). A Lactobacillus-derived biosurfactant inhibits biofilm formation of human pathogenic Candida albicans biofilm producers. Appl Microbiol Biotechnol, 2, 827-837.

27. Resende, A. H. M., Farias, J. M., Silva, D. D., Rufino, R. D., Luna, J. M., Stamford, T. C. M., \& Sarubbo, L. A. (2019). Application of biosurfactants and chitosan in toothpaste formulation. Colloids and Surfaces B Biointerfaces, 181, 77-84.

28. Tambone, E., Bonomi, E., Ghensi, P., Maniglio, D., Ceresa, C., Agostinacchio, F., ... \& Tessarolo, F. (2020). Rhamnolipid Coating Reduces Microbial Biofilm Formation on Titanium Implants: an invitro Study.

29. Kuyukina, M. S., Ivshina, I. B., Baeva, T. A., Kochina, O. A., Gein, S. V., \& Chereshnev, V. A. (2015). Trehalolipid biosurfactants from nonpathogenic Rhodococcus actinobacteria with diverse immunomodulatory activities. New biotechnology, 32(6), 559-568.

30. Burket, L.W., Greenberg, M.S., Glick, M. (2003). Burket's Oral Medicine: Diagnosis and Treatment. Hamilton, $6^{\text {th }}$ edition.

31. Vollenbroich, D., Özel, M., Vater, J., Kamp, R. M., \& Pauli, G. (1997). Mechanism of inactivation of enveloped viruses by the biosurfactant surfactin from Bacillus subtilis. Biologicals, 25(3), 289-297.

32. Naruse, N., Tenmyo, O., Kobaru, S., Kamei, H., Miyaki, T., Konishi, M., \& Oki, T. (1990). Pumilacidin, a complex of new antiviral antibiotics. The Journal of antibiotics, 43(3), $267-$ 280.

33. Shah, V., Doncel, G. F., Seyoum, T., Eaton, K. M., Zalenskaya, I., Hagver, R., ... \& Gross, R. (2005). Sophorolipids, microbial glycolipids with antihuman immunodeficiency virus and spermimmobilizing activities. Antimicrobial agents and chemotherapy, 49(10), 4093-4100.

34. Richards, J. P., Cai, W., Zill, N. A., Zhang, W., \& Ojha, A. K. (2019). Adaptation of Mycobacterium tuberculosis to biofilm growth is genetically linked to drug tolerance. Antimicrobial agents and chemotherapy, 63(11), e01213-19.

35. Clarke, K. G., Ballot, F., \& Reid, S. J. (2010). Enhanced rhamnolipid production by Pseudomonas aeruginosa under phosphate limitation. World 
Journal of Microbiology and Biotechnology, 26(12), 2179-2184.

36. Tahmourespour, A., Kasra-Kermanshahi, R., \& Salehi, R. (2019). Lactobacillus rhamnosus biosurfactant inhibits biofilm formation and gene expression of caries-inducing Streptococcus mutans. Dental research journal, 16(2), 87.

37. Nielsen, T. H., Sørensen, D., Tobiasen, C., Andersen, J. B., Christophersen, C., Givskov, M., \& Sørensen, J. (2002). Antibiotic and biosurfactant properties of cyclic lipopeptides produced by fluorescent Pseudomonas spp. from the sugar beet rhizosphere. Applied and environmental microbiology, 68(7), 3416-3423.

38. Joshi-Navare, K., \& Prabhune, A. (2013). A biosurfactant-sophorolipid acts in synergy with antibiotics to enhance their efficiency. BioMed research international, 2013.

39. Bharali, P., Saikia, J. P., Ray, A., \& Konwar, B. K. (2013). Rhamnolipid (RL) from Pseudomonas aeruginosa OBP1: a novel chemotaxis and antibacterial agent. Colloids and Surfaces B: Biointerfaces, 103, 502-509.

40. Saravanakumari, P., \& Mani, K. (2010). Structural characterization of a novel xylolipid biosurfactant from Lactococcus lactis and analysis of antibacterial activity against multi-drug resistant pathogens. Bioresource technology, 101(22), 88518854.

41. Cochis, A., Fracchia, L., Martinotti, M. G., \& Rimondini, L. (2012). Biosurfactants prevent in vitro Candida albicans biofilm formation on resins and silicon materials for prosthetic devices. Oral surgery, oral medicine, oral pathology and oral radiology, 113(6), 755-761.

42. Kuo, C. H., Lin, Y. W., \& Chen, R. S. (2015). Lipopeptides extract from Bacillus amyloliquefaciens induce human oral squamous cancer cell death. Asian Pacific journal of cancer prevention. Asian Pac J Cancer Prev, 16, 91-96.

43. Shao, L., Song, X., Ma, X., Li, H., \& Qu, Y. (2012). Bioactivities of sophorolipid with different structures against human esophageal cancer cells. Journal of Surgical Research, 173(2), 286291.

44. Donio, M. B. S., Ronica, F. A., Viji, V. T., Velmurugan, S., Jenifer, J. S. C. A., Michaelbabu, M., ... \& Citarasu, T. (2013). Halomonas sp. BS4, A biosurfactant producing halophilic bacterium isolated from solar salt works in India and their biomedical importance. SpringerPlus, 2(1), 149.

45. Christova, N., Lang, S., Wray, V., Kaloyanov, K., Konstantinov, S., \& Stoineva, I. (2015). Production, structural elucidation and in vitro antitumor activity of trehalose lipid biosurfactant from Nocardia farcinica strain. J. Microbiol. Biotechnol, 25(4), 439-447.

46. Thomas, C. E., Ehrhardt, A., \& Kay, M. A. (2003). Progress and problems with the use of viral vectors for gene therapy. Nature Reviews Genetics, 4(5), 346-358.

47. Maitani, Y., Yano, S., Hattori, Y., Furuhata, M., \& Hayashi, K. (2006). Liposome vector containing biosurfactant-complexed DNA as herpes simplex virus thymidine kinase gene delivery system. Journal of Liposome Research, 16(4), 359372.

48. Faivre, V., \& Rosilio, V. (2010). Interest of glycolipids in drug delivery: from physicochemical properties to drug targeting. Expert Opinion on Drug Delivery, 7(9), 1031-1048.

49. Fopase, R., Pathode, S. R., Sharma, S., Datta, P., \& Pandey, L. M. (2020). Lipopeptide and essential oil based nanoemulsion for controlled drug delivery. Polymer-Plastics Technology and Materials, 59(18), 2076-2086.

50. Zhao, H., He, W., Wang, Y., Yue, Y., Gao, X., Li, Z., \& Zhang, X. (2008). Biomineralizing synthesis of mesoporous hydroxyapatite-calcium pyrophosphate polycrystal using ovalbumin as biosurfactant. Materials Chemistry and Physics, 111(2-3), 265-270. 